\title{
Nuclear Emulsion Technology and Directional Dark Matter Study
}

\section{Tatsuhiro Naka*}

Kobayashi-Maskawa Institute for the Origin of Particles and the Universe, Nagoya University

Furo-cho, Chikusa, Nagoya, Japan

E-mail: naka@flab.phy.nagoya-u.ac.jp

\section{Takashi Asada}

Graduate School of Science, Nagoya University Furo-cho, Chikusa, Nagoya, Japan

E-mail: asadaeflab.phys.nagoya-u.ac.jp

\section{Takayoshi Katsuragawa}

Graduate School of Science, Nagoya University Furo-cho, Chikusa, Nagoya, Japan

E-mail: katsuragawa@flab.phys.nagoya-u.ac.jp

\section{Masahiro Yoshimoto}

Graduate School of Science, Nagoya University Furo-cho, Chikusa, Nagoya, Japan E-mail: yoshimotodflab.phys.nagoya-u.ac.jp

\section{Atsuhiro Umemoto}

Graduate School of Science, Nagoya University Furo-cho, Chikusa, Nagoya, Japan E-mail: umemotoeflab.phys.nagoya-u.ac.jp

\section{Shunji Furuya}

Graduate School of Science, Nagoya University Furo-cho, Chikusa, Nagoya, Japan

E-mail: furuyadflab.phys.nagoya-u.ac.jp

\section{Shogo Machii}

Graduate School of Science, Nagoya University Furo-cho, Chikusa, Nagoya, Japan E-mail: machiieflab.phys.nagoya-u.ac.jp

\section{Mitsuhiro Nakamura}

Graduate School of Science, Nagoya University Furo-cho, Chikusa, Nagoya, Japan E-mail: nakamura@flab.phys.nagoya-u.ac.jp 


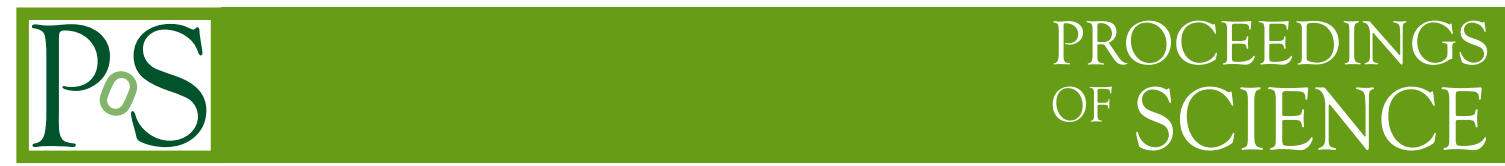

\section{Toshiyuki Nakano}

Graduate School of Science, Nagoya University Furo-cho, Chikusa, Nagoya, Japan

E-mail: nakanodflab.phys.nagoya-u.ac.jp

\section{Osamu Sato}

Graduate School of Science, Nagoya University Furo-cho, Chikusa, Nagoya, Japan

E-mail: sato@flab.phys.nagoya-u.ac.jp

For the latest nuclear emulsion technology, the key technologies are an automatic readout and a self-production system for the nuclear emulsion device. In terms of the readout system, the 100 times higher scanning power than current one is developed by mount of very wide field lens and 72 censers to cover that. In terms of the self-production system of the device, practicable devices have been produced with the crystal sizes from $20 \mathrm{~nm}$ to $200 \mathrm{~nm}$. In this system, the nuclear emulsion of $1 \mathrm{~kg}$ per week can be produced. Fine-grained nuclear emulsion is a quite new detector produced here, and we proposed the directional dark matter search with that. By using this, we can detect the nuclear recoiled tracks more than $100 \mathrm{~nm}$ with direction sensitivity. And, by new optical microscope and X-ray microscope system, such very short length tracks are read out with better than angular resolution of 30 degrees. These systems were demonstrated by the events induced by fast neutrons, and we could see the clear angular distribution with anisotropy. Now, we are promoting the study about the understanding of backgrounds, upgrade of the technologies and underground handling collaborating with Italian scientists.

KMI International Symposium 2013 on "Quest for the Origin of Particles and the Universe", 11-13 December, 2013

Nagoya University, Japan

\footnotetext{
* Speaker.
} 


\section{Introduction}

\subsection{Nuclear Emulsion}

Nuclear Emulsion is a kind of photographic film, and it is capable of detecting the charged particles as tracks with 3D informations and very high spatial resolution. The detection principle of charged particles is as follow. Nuclear emulsion is composed by silver halide crystals (usual size is about $200 \mathrm{~nm}$ ) dispersing in the gelatin. When a charged particle is running in the emulsion film, it should penetrate the crystals. Then, latent image specks are formed on each crystals by ionized electrons and interior silver ions in the crystal due to following reaction,

$$
\begin{gathered}
\mathrm{e}^{-}+\mathrm{Ag}^{+} \rightarrow \mathrm{Ag} \\
\mathrm{e}^{-}+\mathrm{Ag}^{+}+\mathrm{Ag} \rightarrow \mathrm{Ag}_{2} \cdots \mathrm{Ag}_{n}
\end{gathered}
$$

Eventually, the latent image specks grow up to visible size throughout a chemical development treatment as line of silver grains. Here, the development possibility depends on the number of $n$ in the above reaction. As the ionized electrons are closed in a crystal, the spatial resolution of the nuclear emulsion can be achieved the crystal size scale $(0.1-1 \mu \mathrm{m})$. This is the reasons why this detector has such entirely high spatial resolution.

\subsection{Automatic Readout System}

Automatic readout for the tracks recorded in the nuclear emulsion is one of the most important technologies because that power involves the detector scale or experimental accuracy. Automatic readout system has been developed since 1983. Current system is called S-UTS, and the speed of scanning is about $40 \mathrm{~cm}^{2}$ per hour for the thickness of $40 \mu \mathrm{m}$. The systems are running in the OPERA experiment which is the largest experiment with nuclear emulsion detector for the appearance search of the neutrino oscillation. However, further system with higher scanning speed (Hyper Track Selector: HTS) is now developed in parallel. This will achieve the speed of 100 times higher than S-UTS by using the very wide field lens with field of view (FOV) of $5 \times 5 \mathrm{~mm}^{2}$ and the 72 censers to cover such wide FOV. We expect the larger scale experiments, higher speed data taking or the analysis using the big data of the large amount of tracks recorded in the nuclear emulsion film will be achieved by this system.

\subsection{Self-production System of Nuclear Emulsion Device}

The Nuclear emulsion has been produced by photographic companies (e.g. Fuji Film, Irford, Kodak) until 2010. However, we constructed the self-production system from the requirement of more flexible R\&D for various control of the silver halide crystal size and the sensitivity for the crystal, because the crystal sizes depend on the spatial resolution and sensitivity, and the sensitivity control of the crystal should be important for the background rejection and the detection efficiency of signals. This system is possible to control the crystal size by high accuracy addition speed control of $\pm 1 \mathrm{ml} / \mathrm{min}$, temperature control and other parameter tuning. Electron microscope image of the silver halide crystals produced at the Nagoya University was show in Fig 1 Such crystal size control methods have been confirmed, and the next step is a study of sensitivity control. Current production scale of this system is about $1 \mathrm{~kg}$ per week. 


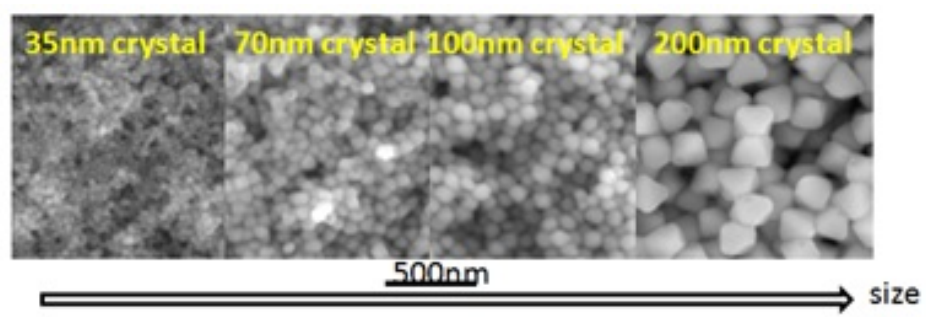

Figure 1: Electron microscope image of the silver halide crystals produced by the self-production system in Nagoya University.

\section{Dark Matter}

A Dark Matter Problem is one of the most important subjects for nature science. Existence of dark matter has been understood in global and local scale for the universe. In the global scale, various observation, simulation and theoretical model (e.g. SDSS[1], CMB [2], N-body simulations) have supported the requirement of dark matter, and for the local scale, anomaly of rotation velocity curve for a galaxy can be interpreted as existence of dark matter around there. Especially, the anomaly has been observed in Milky Way Galaxy[3], and recently, high precision measurement by a VERA experiment [4] updated the rotation velocity around solar system, the result still support the existence of dark matter around the solar system more strongly.

\subsection{Situation of Direct Dark Matter Search}

We can estimate the local dark matter density around solar system from the result of the rotation velocity curve of the Milky Way Galaxy. The estimated value is about $0.3-0.5 \mathrm{GeV} / \mathrm{cm}^{3}$. This value means what we can detect the dark matter directly using dark matter and target nuclei scattering on the earth if the cross section has the detectable range for our technology. Finally, the dark matter signals should have the annual modulation accompanying the revolution of the earth surround the sun.

From the last decade, various dark matter search experiments are running in the world, and their groups are aiming to observe the annual modulation signal finally. In those, DAMA/LIBRA experiments 5 in the Gran Sasso National laboratory in Italy (LNGS) claimed the positive results with very clear annual modulation with $9 \sigma$ significance, and the modulation still continue from 1996 (DAMA/NaI) to 2013. For example, CoGenT[6], CRESST 《7]and CDMS匹8 groups also claimed the positive results in the past few years. Their cross sections for dark matter and nucleon interaction have around $10^{-40}-10^{-42} \mathrm{~cm}^{2}$ area (spin-independent interaction). However, other experiments (e.g. XENON experiments[9]) are excluding those regions. Now, such positive and negative results are mixed in this field.

\subsection{Directional Dark Matter Search}

We advocate that the search with different information should be important to overcome such the chaotic situations in the direct dark matter search field, and the candidate method is a directional dark matter search[10]. This method aims the detection of anisotropy of angular distribution 
because the direction of dark matter is expected to come from Cygnus direction due to rotating of the solar system to the galaxy toward that. If we can detect the dark matter wind using the direction sensitive detector, the existence of dark matter can be indicated with high reliability.

The standard approach for the directional search is to detect the nuclear recoil signals as tracks and to observe the anisotropy of angular distribution. Tracking technology for such low energy atom is one of the challenging subjects for this motivation.

\subsection{Directional Dark Matter Search with Nuclear Emulsion}

Nuclear emulsion is a solid detector of density of about $3 \mathrm{~g} / \mathrm{cm}^{3}$, therefore the expected track length should be less than $1 \mu \mathrm{m}$ for expected the dark matter velocity. For the detection of such very short length tracks, the fine-grained nuclear emulsion is required because the micronization of the silver halide crystals means to increase the spatial resolution. Study of fine-grained nuclear emulsion technology is entirely new in the nuclear emulsion history. In parallel, various specialized new technologies for that (e.g., readout technology for such short length tracks, background rejection, sensitivity control) are required The sensitivity aimed in spin-independent interaction is $10^{-42}-10^{-43} \mathrm{~cm}^{2}$ for the dark matter mass of more than $10 \mathrm{GeV} / \mathrm{c}^{2}$ region with several $10 \mathrm{~kg}$ detector.

\subsubsection{Fine-grained Nuclear Emulsion Device}

The detection of submicron length track is required, and such very short length tracks cannot be detected by the usual nuclear emulsion film because the crystal size is too large. Therefore the silver halide crystals have to be the size of less than $40 \mathrm{~nm}$ [11]. We have already confirmed the method to control the crystal sizes, and there are two type of prototypes with crystal size of about $40 \mathrm{~nm}$ (Nano Imaging Tracker : NIT) and about $20 \mathrm{~nm}$ (Ultra-Nano Imaging Tracker : U-NIT). The crystal size distributions are shown in Fig 2 Such fine-grained nuclear emulsions were confirmed to be capable of detecting the submicron length tracks using ion-implant system. The electron microscope image for the $\mathrm{Kr}$ ion tracks of $200 \mathrm{keV}$ and $400 \mathrm{keV}$ were shown in Fig 3 Detectable track length is more than 200nm for NIT and $100 \mathrm{~nm}$ for U-NIT, and these correspond to $80 \mathrm{keV}$ and $40 \mathrm{keV}$ of $\mathrm{C}$ ion energy respectively because $\mathrm{C}(\mathrm{N}, \mathrm{O})$ are essential target for the dark matter search with higher sensitivity.

\subsubsection{Readout Technology for Submicron Tracking}

Development of readout for the submicron length tracks is one of the challenging subjects in this project. First, an optical microscope scanning is required for the large volume readout because it can achieve the high scanning speed. However, the optical resolution makes the identification of signals hard. Actually, usual optical resolution is following the Rayleigh Criterion, then typical resolution is about $250 \mathrm{~nm}$. However, optical microscopy can work as a roughly candidate selection tool because the signals with more than $200 \mathrm{~nm}$ length at least can be distinguished as an elliptical shape. For example, elliptical fitting with image processing technology is expected to be the available parameter to select the candidates [12].

However, as selected candidate events have to be confirmed as line of silver grains, optical resolution is not enough to do that. Therefore we introduced the combined system with a higher 

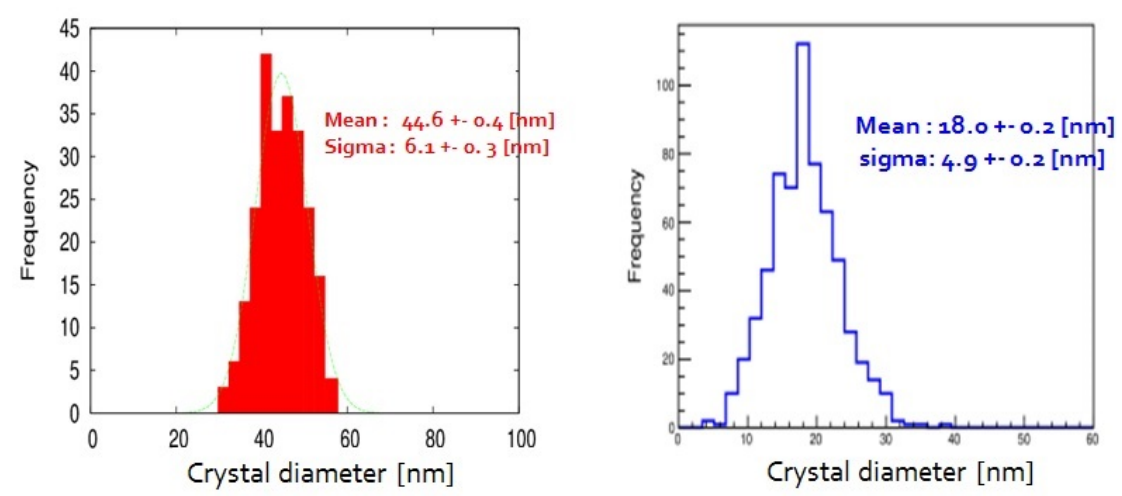

Figure 2: Silver halide crystal size distributions for the fine-grained nuclear emulsion produced in the Nagoya University measured by electron microscope. (a) Nano Imaging Tracker, (b) Ultra-Nano Imaging Tracker.

(a)

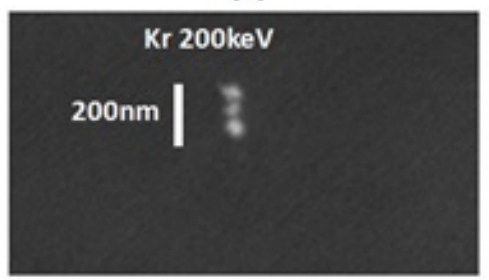

(b)

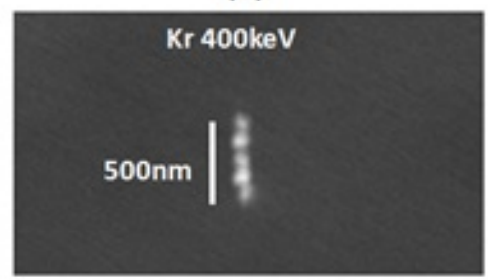

Figure 3: Electron microscope images of typical $\mathrm{Kr}$ ion tracks (a) $200 \mathrm{keV} \mathrm{Kr}$ ion, (b) $400 \mathrm{keV} \mathrm{Kr}$ ion

resolution microscope. The good candidate is a hard X-ray microscope. The development of the $\mathrm{X}$-ray microscope has been advanced by collaboration with scientists with SPring-8 (JASRI), Japan [13]. Advantage of this system is to be able to achieve the non-destructive and high resolution observation. As actual optics, X-ray energy of 6 or $8 \mathrm{keV}\left(\Delta \mathrm{E} / \mathrm{E} \sim 10^{-4}\right)$, zone plate (outer most zone width of $50 \mathrm{~nm}$ ) as focusing lens are utilized. In addition, high contrast imaging could be achieved by the phase contrast method using Zernike phase plate. Spatial resolution was evaluated by the line and space of Ta (Ta thickness of $100 \mathrm{~nm}$ ). This system has the spatial resolution better than $70 \mathrm{~nm}$, and this value is consistent with theoretical value.

\subsubsection{Demonstration of Readout for Submicron Length Tracks}

As a realistic demonstration for direct dark matter search, we tested the readout system using the nuclear recoiled events induced by fast neutrons. Here, heavy nuclei targets (mostly $\mathrm{Br}$ recoil) were used because of neutron energy of $14 \mathrm{MeV}$ due to D-T nuclear fission reaction. First demonstration is to select the candidate events using optical microscope readout system, and to confirm those candidate events using X-ray microscope as next step. These processes are possible by using the fiducial marks to define the coordinate and affine parameter between both microscope systems. The example of the results in this test using both systems is shown in Fig 4 In terms of current system, the matching efficiency of about $100 \%$ and position accuracy of better than $5 \mu \mathrm{m}$ were 
achieved.

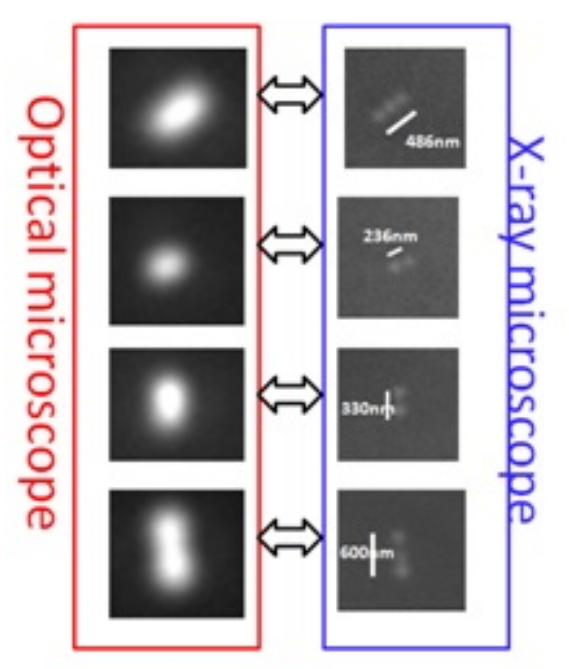

Figure 4: Optical and X-ray microscope images of submicron length tracks induced by neutrons. The left figures are the candidate events selected by optical microscope readout, and the right figures are the confirmed events observed by X-ray microscope.

In contrast, by going through the reverse process from X-ray microscope to optical microscope observation, we can evaluate the selection efficiency for optical readout with non-bias. Major and minor length distribution for elliptical fitting with optical microscopy to the events already confirmed by X-ray microscope was shown in Fig 5 a). Here, blue $(\bigcirc)$ events are random noises, and red (+) events are clear submicron length tracks. This result indicates that the elliptical parameter is a good method to define the signal region for selection of the candidate events by optical microscope readout, but the level depends on the spatial resolution of optical microscope and backgrounds level.

Finally, the angular distribution for the events confirmed as tracks was shown in Fig 5 b). Here, angle of $0 \mathrm{deg}$. is corresponds to the direction incoming the neutrons. Therefore we could see the directionality of recoiled nuclei with submicron length. Current angular resolution of the detector is better than 30 degrees for $200 \mathrm{~nm}$ length from the evaluation using ion-implant system.

\subsection{Underground Activity}

Now, we are promoting the underground activity toward the underground experiment. In the National Laboratory of Gran Sasso (LNGS) at Italy, the small facility for the R\&D of handling of detector have been constructed in hall B. Simultaneously, low-radioactivity measurements are progressed by ICP-MS (Inductively Coupled Plasma - Mass Spectrometer) and Ge spectroscopy. In terms of the study to understand the backgrounds, the neutron flux measurement project are also planning because the nuclear emulsion should work well as fast neutron detector by detection of recoiled protons induced by neutron. The nuclear emulsion device for the neutron measurement has no challenging subject, and main subject is the method of readout technology and understanding the backgrounds. Therefore, this measurement should be able to be started more promptly. Finally, 
(a)

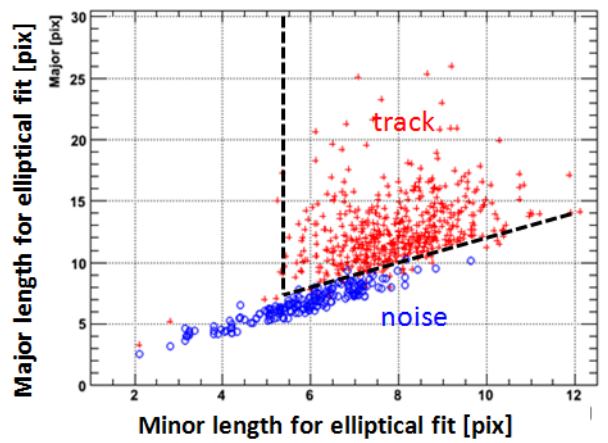

(b)

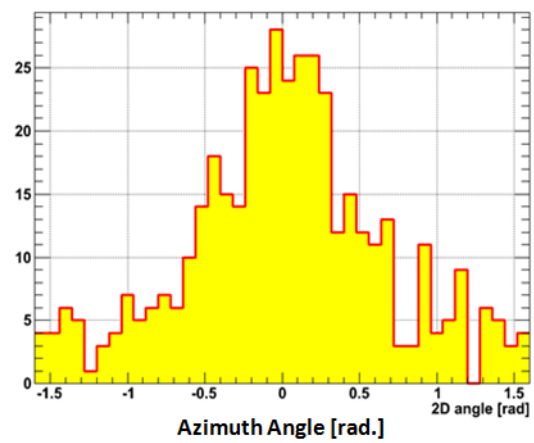

Figure 5: The result of optical microscope analysis for that events confirmed by X-ray microscope. (a) Major and minor length distribution for elliptical fitting in the optical microscopy. Blue $(\bigcirc)$ events are non-track events, and red events $(+)$ are submicron length events. (b) angular distribution for the red events.

we aim the measurement whether the fast neutron flux in the underground has annual modulation or not. These activities are going by collaboration with Italian scientists.

\subsection{Summary and Future Planning}

Directional dark matter search should be great interest as the method for the direct dark matter search in the next generation because the current situation hope the another information to understand the signal and backgrounds. Quite high spatial resolution for the fine-grained nuclear emulsion enable the detection of nuclear recoil signals correspond to the energy for the dark matter detection as tracks. In this study, by development of new readout technology using the epiilluminated optical microscope and hard X-ray microscope system, very short length tracks with submicron could be read out automatically.

As next step, we have to evaluate the response of detector for the absolute efficiency and background level and study about the low-background detector (i.e., insensitive emulsion to the gamma or beta-rays).

This work was supported by JSPS Grant-in-Aid for Young Scientists (B) (25800140) and the synchrotron radiation experiments were performed at the BL37 and 47 in the SPring- 8 with the approval of the Japan Synchrotron Radiation Research Institute (JASRI).

\section{References}

[1] Max Tegmark et al., Cosmological parameters from SDSS and WMAP, Phys. Rev. D 69, 103501 (2004)

[2] G. Hinshaw et al., Nine-year Wilkinson Microwave Anisotropy Probe (WMAP) Observations: Cosmological Parameter Results, ApJS 208 (1013) 19H

[3] Clemens et al., Atrophys. J 422 (1985) 295

[4] Toshihiro Omodaka, VERA Project: Measuring Our Milky Way Galaxy, AAPPS Bulletin 19 (2009) 3 
[5] R. Bernabei et al., New Result from DAMA/LIBRA, Eur. Phys. J C 67 (2010) 39 //DAMA

[6] C. E. Aalseth et al., Serach for an Annual Modulation in a p-Type Point Contact Germanium DarkMatter Detector, Phys. Rev. Lett. 107 (2011) 141301

[7] G. Angloher et al., Result from 730 kg days of the CRESST-âĔąDark Matter search, Eur. Phys. J 72 (2012) 1971

[8] R. Agnese et al., Silicon Detector Dark Matter Results from the Final Exposure of CDMSăĚa, arXiv:1304.4279v3 [hep-ex] (2013)

[9] E. Aprile et al. , Dark Matter Results from 225 Live Days of XENON100 Data, Phys. Rev. Lett 109 (2012) 181301

[10] D.N. Spergel, Mothion of the Earth and the detection of weakly interacting massive particles, Phys. Rev. D 37 (1988) 1353

[11] M. Natsume et al., Low-volocity ion tracks in fine grain emulsion, Nucl. Inst. Meth A 575 (2007) 439

[12] M. Kimura and T. Naka, Submicron track readout in fine-grained nuclear emulsion using optical microscopy, Nucl. Inst . Meth. A 680 (2012) 12

[13] Yoshio Suzuki et al., Development of large-field high-resolution hard x-ray imaging microscopy and microtomography with Fresnel zone plate objective, Proc. Of SPIE 8851 (2013) 885109 